MILENA VISENTAINER ${ }^{1}$

EDWARD ARAUJO JÚNIOR ${ }^{2}$

LIIIAM CRISTINE ROLO 3

LUCIANO MarCondes MaChado NardOZZA ${ }^{4}$

ANTONIO FERNANDES MORON ${ }^{5}$

Artigo original

Palavras-chave

Feto

Corpo caloso

Valores de referência

Ultrassonografia

Imagem tridimensional

Keywords

Fetus

Corpus callosum

Reference values

Ultrasonography

Imaging, three-dimensional

\section{Avaliação do comprimento e área do corpo caloso fetal por meio da ultrassonografia tridimensional}

\author{
Assessment of length and area of corpus callosum by three-dimensional \\ ultrasonography
}

\section{Resumo}

OBJETIVO: determinar os valores de referência para o comprimento e a área do corpo caloso fetal entre a $20^{a}$ e $33^{\circ}$ semanas de gestação por meio da ultrassonografia tridimensional (US3D). MÉTODOS: foi realizado um estudo do tipo corte transversal com 70 gestantes normais entre a $20^{a}$ e $33^{a}$ semanas de gestação. Utilizou-se um aparelho da marca Accuvix $X Q$, equipado com transdutor convexo volumétrico (3 a $5 \mathrm{MHz}$ ). Para a obtenção do corpo caloso fetal, foi utilizado um plano transfrontal, com a sutura metópica como janela acústica. Para o cálculo do comprimento, utilizou-se a distância entre os pontos médios dos polos proximal e distal do corpo caloso. Para o cálculo da área, a delimitação manual da superfície externa do corpo caloso foi realizada. Para o comprimento e a área do corpo caloso, foram calculadas: as médias, as medianas, os desvios padrão e os valores máximo e mínimo. Para a correlação da área e do comprimento do corpo caloso com a idade gestacional e o diâmetro biparietal foram criados diagramas de dispersão, sendo a qualidade dos ajustes verificada pelo coeficiente de determinação $\left(\mathbb{R}^{2}\right)$. Para a variabilidade intraobservador, utilizou-se o coeficiente de correlação intraclasse (CCI). RESULTADOS: a média do comprimento do corpo caloso variou de 21,7 mm (1 8,6 - 25,2 mm) a 38,7 $\mathrm{mm}(32,6-43,3 \mathrm{~mm})$ entre a $20^{a}$ e $33^{a}$ semanas, respectivamente. A média da área do corpo caloso variou de 55,2 mm² $\left(41,0-80,0 \mathrm{~mm}^{2}\right)$ a $142,2 \mathrm{~mm}^{2}\left(114,0-160,0 \mathrm{~mm}^{2}\right)$ entre a $20^{\circ}$ e $33^{a}$ semanas, respectivamente. $O$ comprimento e a área do corpo caloso foram fortemente correlacionados com a idade gestacional ( $R^{2}=0,7$ e 0,7 , respectivamente) e com o diâmetro biparietal $\left(R^{2}=0,7\right.$ e 0,6, respectivamente). A variabilidade intraobservador foi adequada com $C C l=0,9$ e 0,9 para o comprimento e área, respectivamente. CONCLUSÕES: valores de referência para o comprimento e área do corpo caloso fetal entre a $20^{a}$ e $33^{a}$ semanas foram determinados. A variabilidade intraobservador foi adequada.

\section{Abstract}

PURPOSE: to establish reference values for the length and area of the fetal corpus callosum between the $20^{\text {th }}$ and $33^{\text {rd }}$ weeks of gestation using three-dimensional ultrasound (3DUS). METHODS: this cross-sectional study involved 70 normal pregnancies with gestational age between 20 and 33 weeks. An Accuvix XQ instrument with a convex volumetric transducer ( 3 to 5 $\mathrm{MHz}$ ) was used. To assess the corpus callosum, a transfrontal plane was obtained using the metopic suture as an acoustic window. Length was obtained by measuring the distance between the proximal and distal extremities of the corpus callosum. Area was obtained by manual tracing of the external corpus callosum surface. The means, medians, standard deviations, and maximum and minimum values were calculated for the corpus callosum length and area. Scatter graphs were created to analyze the correlation between corpus callosum length and area and gestational age and biparietal diameter, the quality adjustments was verified according to the determination coefficient $\left(\mathrm{R}^{2}\right)$. The intraclass correlation coefficient (ICC) was used to assess the intraobserver variability. RESULTS: mean corpus callosum length increased from 21.7 (18.6-25.2 mm) to 38.7 $\mathrm{mm}(32.6-43.3 \mathrm{~mm})$ between 20 and 33 weeks of pregnancy, respectively. Mean corpus callosum area increased from $55.2\left(41.0-80.0 \mathrm{~mm}^{2}\right)$ to $\left.142.2 \mathrm{~mm}^{2}(1) 14.0-160.0 \mathrm{~mm}^{2}\right)$, between 20 to 33 weeks of pregnancy, respectively. There was a strong correlation between corpus callosum length and area and gestational age $\left(R^{2}=0.7\right.$ and 0.7 , respectively) and biparietal diameter ( $R^{2}=0.7$ and 0.6 , respectively). Intraobserver variability was appropriate, with an ICC of 0.9 and 0.9 for length and area, respectively. CONCLUSIONS: reference values for corpus callosum length and area were established for fetuses between 20 and 33 weeks gestation. Intraobserver variability was appropriate.
Correspondência:

Edward Araujo Júnior Departamento de Obstetríicia da Universidade Federal de São Paulo -

UNIFESP

Rua Napoleão de Barros, 875 - Vila Clementino CEP: 04024-002 - São Paulo (SP), Brasi

Tel./Fax: (11) 5571-076

E-mail: araujoired@terra.com.br

Recebido

$26 / 07 / 10$

Aceito com modificacões

$12 / 11 / 10$
Setor de Neurologia Fetal do Departamento de Obstetrícia da Universidade Federal de São Paulo - UNIFESP, São Paulo (SP), Brasil.

'Pós-graduanda (Mestrado) do Departamento de Obstetrícia da Universidade Federal de São Paulo - UNIFESP - São Paulo (SP), Brasil.

2 Professor Adjunto do Departamento de Obstetrícia da Universidade Federal de São Paulo - UNIFESP - São Paulo (SP), Brasil.

${ }_{3}^{3}$ Mestre e Pós-graduanda (Doutorado) do Departamento de Obstetrícia da Universidade Federal de São Paulo - UNIFESP - São Paulo (SP), Brasil.

4 Professor Adjunto e Chefe da Disciplina de Medicina Fetal do Departamento de Obstetrícia da Universidade Federal de São Paulo - UNIFESP - São Paulo (SP), Brasil.

5 Professor Titular do Departamento de Obstetrícia da Universidade Federal de São Paulo - UNIFESP - São Paulo (SP), Brasil. 


\section{Introdução}

O corpo caloso (CC) é a maior junção entre os hemisférios cerebrais, estendendo-se do lobo frontal anteriormente até abaixo da placa quadrigêmea, posteriormente ${ }^{1}$. A sua formação (anterior para posterior) é iniciada no período embrionário e completada entre 18 a 20 semanas de gestação ${ }^{2}$.

Durante o desenvolvimento intrauterino, a presença de disgenesias do CC coincide com outras malformações cerebrais em $80 \%$ dos casos, inclusive síndromes neurogenéticas ${ }^{3}$. A incidência de anormalidades do CC (hipoplasia, agenesia total e parcial) varia de 0,3 a $0,7 \% 4$. O quadro clínico que indique anomalia do CC não é específico, sendo o diagnóstico feito, na maior parte das vezes, em exames ocasionais. A maioria dos eventuais sintomas está relacionada com as outras malformações do sistema nervoso central (SNC), as quais coexistem com as disgenesias do CC. Os sintomas mais frequentes são: atraso de desenvolvimento neuropsicomotor, convulsões ou movimentos incoordenados 5 .

Durante o pré-natal, a ultrassonografia bidimensional (US2D) pode diagnosticar, ou pelo menos sugerir, disgenesia ou agenesia do CC. Utilizando-se um plano médio sagital do cérebro, o CC normal aparece como estrutura fina, curvilínea e hipoecóica que forma o teto do cavo do septo pelúcido, dos cornos anteriores e do corpo dos ventrículos laterais. Contudo, deve-se referir como principal limitação do plano médio sagital, a falta da clara diferenciação do CC em relação à porção inferior do septo pelúcido ${ }^{6}$. Além disso, os casos de hipoplasia e agenesia parcial do CC pela US2D são extremamente difíceis de serem realizados, porque apesar de o CC ser identificado com forma atípica, a forma da cabeça fetal no plano axial é frequentemente normal ${ }^{7}$.

A ultrassonografia tridimensional (US3D) surgiu no início da década de 1990 e apresenta as seguintes vantagens em relação à US2D: possibilidade de análise das estruturas fetais em três planos ortogonais, maior acurácia na medida volumétrica e envio de volumes para serem analisados em centros terciários ${ }^{8}$. Em relação ao SNC fetal, a US3D permite a reconstrução de planos médio-sagitais, possibilitando a visualização do $C^{6,9-11}$. Contudo, as reconstruções geram o chamado "complexo sonoluscente em vírgula" devido à incapacidade de tal reconstrução, assim como na US2D, em discernir claramente o CC do cavo do septo pelúcido. A utilização da sutura metópica como janela acústica permite a obtenção do plano transfrontal para visualização de estruturas da linha média, demonstrando que a visão direta deste plano é superior aos planos sagitais obtidos por reconstruções de aquisições axiais, principalmente, devido a menos artefatos e sombras acústicas ${ }^{12}$.
Recentemente, foi descrita uma nova técnica de obtenção do CC fetal por meio da US3D utilizando a via transabdominal ${ }^{13}$. Por meio de planos sagitais, axiais e coronais, foram obtidos volumes do crânio fetal, tanto em escala de cinzas como com Power Doppler. Por meio da manipulação destes volumes nos três planos ortogonais, foi possível obter imagens de qualidade do CC em 93,1\% dos casos e da artéria pericalosa em 94,4\% ${ }^{13}$.

Devido à importância do diagnóstico pré-natal de agenesias parciais do CC como uma forma de aconselhamento dos pais, associada a uma baixa taxa de detecção à US2D, o objetivo deste estudo é determinar os valores de referências para o comprimento e a área do CC fetal por meio da US3D, utilizando o plano transfrontal.

\section{Métodos}

Foi realizado um estudo do tipo corte transversal com 70 gestantes normais, com idades gestacionais entre a $20^{a}$ e $33^{a}$ semanas, no período de setembro de 2009 a março de 2010. Este estudo foi aprovado pelo Comitê de Ética em Pesquisa da Universidade Federal de São Paulo (UNIFESP), sob o $\mathrm{n}^{\circ} 1.071 / 09$, sendo que as pacientes que concordaram com a participação voluntária assinaram termo de consentimento livre e esclarecido.

Os critérios de inclusão foram: gestação única com feto vivo; idade gestacional (IG) determinada pela data da última menstruação (DUM) se houver ciclos menstruais regulares; nos casos de DUM incerta, se foi utilizada uma datação baseada na ultrassonografia realizada até a $20^{\mathrm{a}}$ semana. Os critérios de exclusão foram: gestações com malformações estruturais detectadas ao exame ultrassonográfico; gestantes portadoras de doenças crônicas (hipertensão crônica, diabetes melito, colagenoses, dentre outras); fetos com peso abaixo do percentil 10 ou acima do percentil 90 para IG, segundo tabela proposta por Hadlock et al. ${ }^{14}$; oligoâmnio (ILA abaixo do percentil 5) ou polidrâmnio (ILA acima do percentil 95), segundo tabela proposta por Moore e Cayle ${ }^{15}$; planos que não permitissem uma avaliação adequada da área e do comprimento do CC. As pacientes eram provenientes do ambulatório de pré-natal de baixo risco do Departamento de Obstetrícia da UNIFESP e/ou de unidades básicas de saúde da cidade de São Paulo, sendo selecionadas de forma aleatória e sem controle de amostragem. Estas pacientes foram avaliadas uma única vez (corte transversal), não se obtendo os seus resultados perinatais.

Os exames foram realizados no Setor de US3D do Departamento de Obstetrícia da UNIFESP, sendo estes de responsabilidade de um único examinador (MV) com experiência de dois anos em US3D em Obstetrícia. Os exames foram executados em um aparelho Accuvix XQ (Medison, Seoul, Korea) equipado com transdutor convexo volumétrico multifrequencial (3-5 MHz). Inicialmente, 
foi desenvolvida uma avaliação bidimensional de forma a serem determinados: a morfologia fetal, a biometria fetal, a quantificação de volume do líquido amniótico e a predição de peso fetal. De forma a se obter um melhor contraste da imagem no modo B, foi ativada a tecla DMR (Dynamic Magnetic Resonance). Posteriormente, foi realizada a avaliação tridimensional, para tanto foi padronizado um ângulo de abertura de $30^{\circ}$, modo de velocidade normal, alta qualidade (high definition). Ativou-se a tecla 3D aparecendo uma janela (BOX) de forma a englobar todo o crânio fetal (ROI - região de interesse). O plano de captura consistiu de uma visão transfrontal, tendo como janela acústica a sutura metópica, segundo padronizado por Vinãls et al. ${ }^{12}$. Após a captura do bloco, a imagem foi apresentada na forma de três planos ortogonais perpendiculares (axial, sagital e coronal), sendo utilizado o plano sagital (plano de aquisição) como referencial. Todas as análises foram feitas na ausência da paciente (off-line) com o uso do software SonoView Pro, versão 1.03 (Medison, Seoul, Korea). Para o cálculo do comprimento do CC, foi selecionada a tecla MESURE, sendo os calipers posicionados nos pontos médios dos polos proximal (anterior) e distal (posterior) do CC. Para o cálculo da área, foi selecionada novamente a tecla MESURE, sendo a superfície externa do CC delimitada de forma manual.

Os dados foram coletados em uma planilha do programa Excel 2003 (Microsoft, Redmond, WA, USA) e analisados por meio do programa estatístico Statistical Package for the Social Sciences (SPSS), versão 13.0 (SPSS, Chicago, IL, USA). As variáveis foram submetidas ao teste Kolmogorov-Smirnov para verificar distribuição normal. Para o comprimento e a área, foram calculadas: as médias, as medianas, os desvios padrão e os valores máximo e mínimo. Para avaliar a correlação do comprimento e área do CC com a IG e diâmetro biparietal (DBP), bem como do DBP com a IG, foram realizadas regressões polinomiais, sendo a qualidade dos ajustes verificada pelo coeficiente de determinação $\left(\mathrm{R}^{2}\right)$. Para o cálculo da variabilidade intraobservador, o mesmo examinador realizou uma segunda medida dos mesmos 70 casos, uma semana após a primeira medida. Para este cálculo, o coeficiente de correlação intraclasse (CCI) e os gráficos de Bland e Altman ${ }^{16}$ foram utilizados. Os gráficos de Bland-Altman plotam as médias das duas medidas, realizadas por um mesmo examinador, contra a diferença de suas médias com intervalo de $95 \%$ de confiança e 1,96 desvios padrão (DP) da média (limites de concordância). Em todas as análises será utilizado nível de significância (p) de 0,05.

\section{Resultados}

A amostra final constituiu-se de 70 gestantes que preencheram os critérios de inclusão e exclusão. A idade materna média foi de 27,9 anos (DP \pm 7,8 anos), o número de gestações variou de um a dois com média de 1,6 , enquanto que a paridade variou de zero a três com média de 0,5 partos.

A média do comprimento do CC variou de 21,7 \pm $1,75 \mathrm{~mm}(18,6-25,2 \mathrm{~mm})$ na $20^{\mathrm{a}}$ para $38,7 \pm 3,9 \mathrm{~mm}$ $(32,6-43,3 \mathrm{~mm})$ na $33^{\mathrm{a}}$ semana. A média da área do CC variou de $55,2 \pm 11,5 \mathrm{~mm}^{2}\left(41,0-80,0 \mathrm{~mm}^{2}\right)$ na $20^{a}$ para $142,2 \pm 17,4 \mathrm{~mm}^{2}\left(114,0-160,0 \mathrm{~mm}^{2}\right)$ na $33^{\mathrm{a}}$ semana. As Tabelas 1 e 2 apresentam as médias, medianas, DP e valores máximo e mínimo para o comprimento e área do CC fetal, respectivamente.

O comprimento do CC foi fortemente correlacionado com a IG, sendo o melhor ajuste com a equação linear: comprimento do $C C=1,4 \times I G-6,8\left(R^{2}=0,7\right)($ Figura 1$)$. Da mesma forma, o comprimento do CC também foi fortemente

Tabela 1 - Análise descritiva do comprimento do corpo caloso fetal em cada intervalo de idade gestacional avaliado

\begin{tabular}{l|ccccc}
\hline Intervalo gestacional & $\begin{array}{c}\text { Média } \\
(\mathbf{m m})\end{array}$ & $\begin{array}{c}\text { Mediana } \\
(\mathbf{m m})\end{array}$ & $\begin{array}{c}\text { Desvio } \\
\text { padrão } \\
(\mathbf{m m})\end{array}$ & $\begin{array}{c}\text { Mínimo } \\
(\mathbf{m m})\end{array}$ & $\begin{array}{c}\text { Máximo } \\
(\mathbf{m m})\end{array}$ \\
\hline 20 a 21 sem 6 dias & 21,7 & 22,0 & 1,7 & 18,6 & 25,2 \\
22 a 23 sem 6 dias & 24,5 & 24,2 & 2,5 & 20,7 & 28,8 \\
24 a 25 sem 6 dias & 28,1 & 28,1 & 2,5 & 22,7 & 33,2 \\
26 a 27 sem 6 dias & 32,0 & 32,5 & 4,1 & 23,2 & 37,4 \\
28 a 29 sem 6 dias & 34,3 & 35,1 & 2,8 & 29,1 & 37,5 \\
30 a 31 sem 6 dias & 35,6 & 36,7 & 4,0 & 28,1 & 40,0 \\
32 a 33 sem 6 dias & 38,7 & 38,6 & 3,9 & 32,6 & 43,3 \\
\hline
\end{tabular}

Tabela 2 - Análise descritiva da área do corpo caloso fetal em cada intervalo de idade gestacional avaliado

\begin{tabular}{|c|c|c|c|c|c|}
\hline Intervalo gestacional & $\begin{array}{l}\text { Média } \\
(\mathrm{mm})\end{array}$ & $\begin{array}{l}\text { Mediana } \\
\text { (mm) }\end{array}$ & $\begin{array}{l}\text { Desvio } \\
\text { padrão } \\
\text { (mm) }\end{array}$ & $\begin{array}{c}\text { Mínimo } \\
\text { (mm) }\end{array}$ & $\begin{array}{c}\text { Máximo } \\
(\mathrm{mm})\end{array}$ \\
\hline 20 a 21 sem 6 dias & 55,2 & 51,0 & 11,5 & 41,0 & 80,0 \\
\hline 22 a 23 sem 6 dias & 73,3 & 71,5 & 24,0 & 39,0 & 118,0 \\
\hline 24 a 25 sem 6 dias & 91,3 & 90,0 & 18,7 & 52,0 & 128,0 \\
\hline 26 a 27 sem 6 dias & 114,8 & 107,0 & 33,4 & 70,0 & 183,0 \\
\hline 28 a 29 sem 6 dias & 128,1 & 114,0 & 27,3 & 109,0 & 176,0 \\
\hline 30 a 31 sem 6 dias & 139,8 & 140,0 & 14,9 & 110,0 & 160,0 \\
\hline 32 a 33 sem 6 dias & 142,2 & 149,0 & 17,4 & 114,0 & 160,0 \\
\hline
\end{tabular}

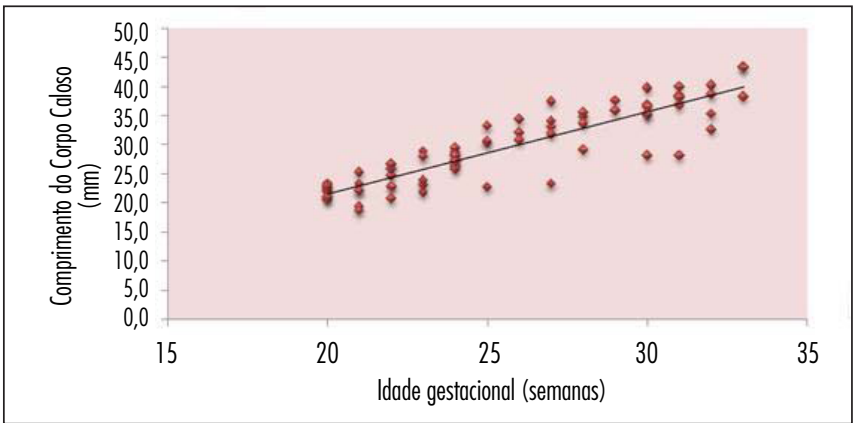

Figura 1 - Gráfico de dispersão do comprimento do corpo caloso fetal em função da idade gestacional. 
correlacionado com o DBP, sendo o melhor ajuste com a equação linear: comprimento do $\mathrm{CC}=0,5 \times \mathrm{DBP}-3,2$ $\left(\mathrm{R}^{2}=0,7\right)$. A área do CC foi fortemente correlacionada com a IG, sendo o melhor ajuste com a equação linear: área do $\mathrm{CC}=7,8 \times \mathrm{IG}-101,5\left(\mathrm{R}^{2}=0,7\right)$. A área do $\mathrm{CC}$ também foi fortemente correlacionada com o DBP, sendo o melhor ajuste com a equação linear: área do $C C=2,8 \mathrm{x}$ DBP - 83,1 $\left(\mathrm{R}^{2}=0,6\right)$. Foi observada uma forte correlação entre o DBP e a IG, sendo o melhor ajuste realizado pela equação exponencial: DBP $=10,4$ e 0,014 x IG, com R ${ }^{2}$ $=0,9$ (Figura 2$)$.

Notou-se adequada variabilidade intraobservador para o comprimento do CC, com CCI $=0,9$ (IC95\% = 0,9; 0,9). O gráfico de Bland-Altman comprova esta boa reprodutibilidade com média das diferenças de $0,2 \mathrm{~mm}$ (IC95\% $\pm 2,8$ e DP $\pm 1,4$ ) (Figura 3). Observou-se também uma adequada variabilidade intraobservador para a área do CC, com CCI = 0,9 (IC95\% = 0,9; 0,9). O gráfico de Bland-Altman comprova esta adequada reprodutibilidade com média das diferenças de $0,04 \mathrm{~mm}^{2}$ (IC95\% $\pm 23,4 \mathrm{e}$ $\mathrm{DP} \pm 11,9)$ (Figura 4).

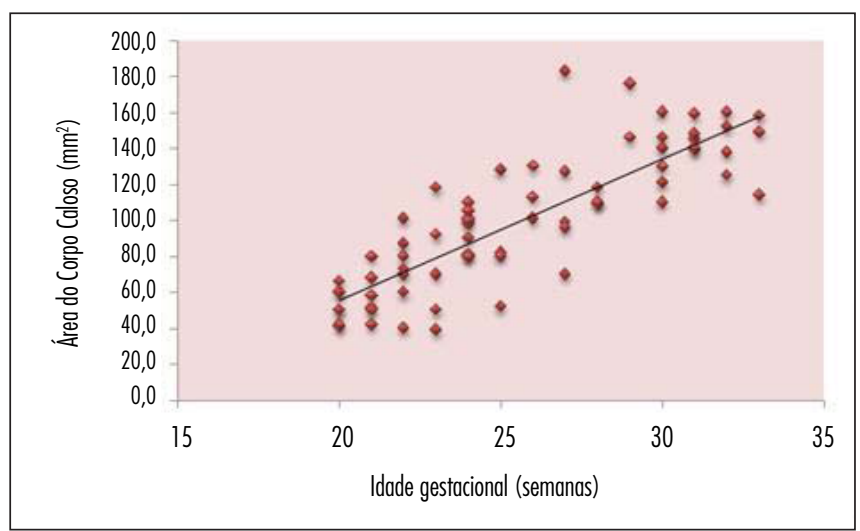

Figura 2 - Gráfico de dispersão da área do corpo caloso fetal em função da idade gestacional.

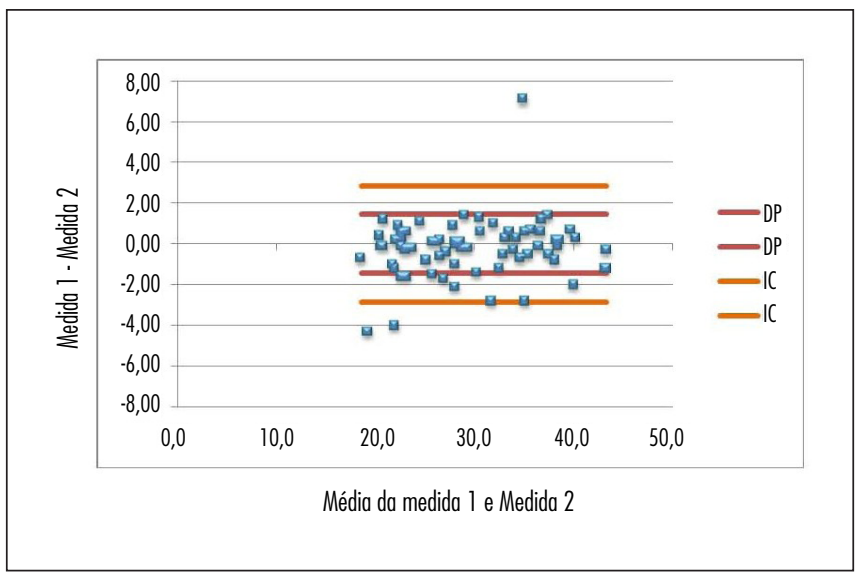

Figura 3 - Diferença das medidas do comprimento do corpo caloso realizadas por um examinador (medida 1 e medida 2) plotadas contra a diferença de suas médias. DP: desvio padrão; IC: intervalo de confiança.

\section{Discussão}

Em humanos, o CC é dividido em cinco regiões. No corte sagital mediano do cérebro, aparece como uma lâmina arqueada dorsalmente, o tronco do CC, que se dilata posteriormente no istmo/esplênio do CC e se flete anteriormente em direção à base do crânio para constituir o joelho do CC. Este se afila para formar o rostro do $\mathrm{CC}$, porção contígua à comissura anterior. Alterações de espessura e volume do CC, em estudos com ressonância magnética de adultos, descrevem as mais variadas correlações com patologias psiquiátricas e neurológicas, tais como: déficit de atenção, hiperatividade, distúrbio bipolar, esquizofrenia e alterações da audição ${ }^{17-19}$.

A US2D é o principal método de diagnóstico prénatal de agenesias parciais ou totais do CC, sendo melhor visualizado no plano médio-sagital como uma estrutura fina, curvilínea e hipoecóica que forma o teto do cavo do septo pelúcido, dos cornos anteriores e do corpo dos ventrículos laterais. Uma tênue linha hiperecóica marca a estrutura superficialmente, em que faz limite com o giro do cíngulo, banda hipoecóica que se estende contiguamente acima do CC. O sulco pericaloso e seus vasos também aparecem como linha mais hiperecóica na interface do CC com o giro do cíngulo, sendo mais facilmente detectado com a insonação do color Doppler, que evidenciará a artéria pericalosa. Já o cavo do septo pelúcido, os cornos anteriores e o corpo dos ventrículos laterais constituem espaços com imagem anecóica abaixo da superfície mais profunda do CC, que também é observada com contorno mais hiperecogênico ${ }^{20,21}$.

Há na literatura apenas três estudos que avaliaram o comprimento do CC por meio da US2D utilizando o plano médio-sagital. No primeiro estudo, os autores realizaram as avaliações por meio da US2D via vaginal para medir o comprimento e a espessura do CC em 101 fetos entre a $18^{\mathrm{a}}$

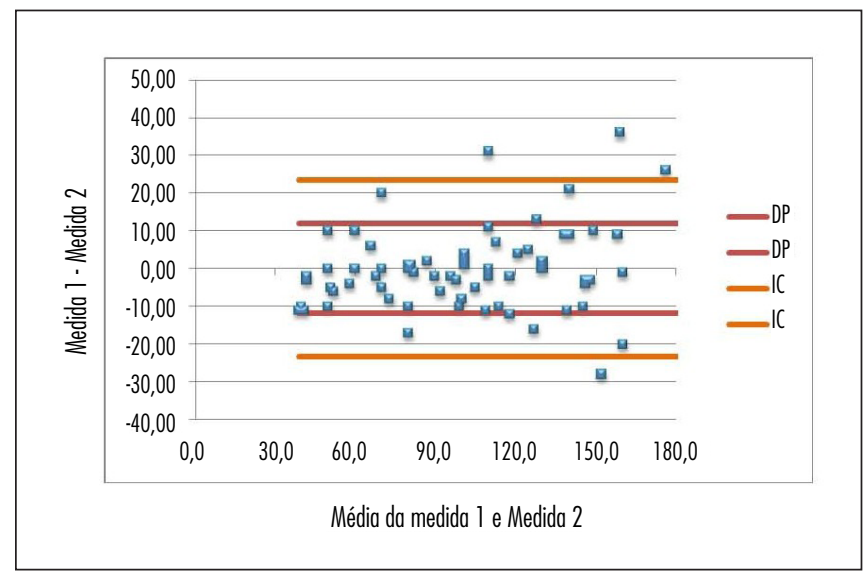

Figura 4 - Diferença das medidas da área do corpo caloso realizadas por um examinador (medida 1 e medida 2) plotadas contra a diferença de suas médias. DP: desvio padrão; IC: intervalo de confiança. 
e $42^{a}$ semanas de gestação. A estrutura pode ser avaliada satisfatoriamente, pelo plano médio sagital, em $95 \%$ dos casos. Foi documentado o aumento em duas vezes da espessura do CC entre a $18^{\mathrm{a}}$ semana e o termo, sendo este crescimento não-linear, alternando períodos de maior e menor ganho. Já o comprimento, inicialmente de $17 \mathrm{~mm}$ entre a $18^{\mathrm{a}}$ e $19^{\mathrm{a}}$ semanas, cresceu para aproximadamente $44 \mathrm{~mm}$ no termo, de forma linear; porém, a relação do comprimento do CC com o diâmetro anteroposterior do crânio passou a ser constante a partir da $20^{a}$ e $21^{a}$ semanas quando o esplênio terminou de se configurar ${ }^{22}$. No segundo estudo, os autores realizaram estudo transversal com 270 gestantes normais entre a $16^{\mathrm{a}}$ e $37^{\mathrm{a}}$ semanas, medindo o comprimento do CC no plano médio-sagital pelas vias vaginal e abdominal. Foi observado, no período entre a $20^{a}$ e $33^{\text {a }}$ semanas, que a média do comprimento do CC variou de 18,9 a $38,5 \mathrm{~mm}^{23}$. Recentemente, em estudo realizado na população chinesa com 622 fetos entre a $16^{a}$ e $39^{a}$ semanas, a média do comprimento do CC variou de 18,7 a $38,2 \mathrm{~mm}^{24}$. Nestes dois últimos estudos, as medidas do comprimento do CC obtidas por planos médiosagitais foram muito semelhantes às no presente estudo, utilizando o plano transfrontal. Neste estudo obtivemos, neste mesmo intervalo gestacional, uma variação de 21,7 a 38,7 mm. Deve-se referir que determinação dos valores de referência para a área do CC fetal determinada neste estudo é inédita na literatura.

Há na literatura um único estudo que utilizou o plano transfrontal, obtido por meio da US3D, na avaliação do comprimento e área do CC fetal. Neste estudo foram avaliadas 60 gestantes, sendo a visão transfrontal obtida em $87 \%$ dos casos. Os autores observaram que o comprimento do CC pela técnica transfrontal foi fortemente correlacionado com aquele obtido pelas técnicas multiplanar $(\mathrm{r}=0,8)$ e volume contrast image c-plane (VCI-C) $(r=0,9)$; contudo, não determinaram valores de referência para este parâmetro ${ }^{12}$. Deve-se referir que a visão transfrontal do CC fetal já havia sido anteriormente descrita por meio da US2D ${ }^{25}$; entretanto, a aquisição por meio da US3D é vantajosa, pois permite correções no modo multiplanar de forma a se obter o plano exato de medida do CC, independentemente de o feto não estar na posição ideal para sua obtenção.

Neste estudo, foi observada forte correlação entre o DBP e o comprimento e área do CC fetal entre a $20^{\mathrm{a}} \mathrm{e}$ $33^{\text {a }}$ semanas, estes resultados demonstram que ambos os parâmetros aumentam de forma similar com a IG. Como o DPB é um importante parâmetro de crescimento do polo cefálico e de datação da IG, a alta correlação observada desse parâmetro com o comprimento e área do CC pode inferir que estes parâmetros também podem se modificar em condições fisiológicas como dólico e braquicefalia, além de condições patológicas como as craniossinostoses. Observou-se ainda uma forte correlação entre o DBP e a IG com $\mathrm{R}^{2}=0,9$, tal resultado mostra-se de grande valor, pois valida as medidas de área e comprimento do CC fetal como parâmetros de normalidade.

Notou-se também, neste estudo, uma adequada reprodutibilidade intraobservador para o comprimento e área do CC fetal utilizando o plano transfrontal por meio da US3D, resultado ainda inédito na literatura. Este resultado se mostra de grande importância, pois valida os valores de referência determinados neste estudo, possibilitando que estes possam ser aplicados em fetos com suspeitas de anormalidades do CC.

Em síntese, este é o primeiro artigo que determinou valores de referência para o comprimento e a área do CC fetal utilizando o plano transfrontal obtido por meio da US3D. Apesar de ser um estudo com limitado número de casos, acredita-se que estes valores possam servir como parâmetro de normalidade a ser aplicado em fetos com suspeita de agenesias total ou parcial do CC, de forma a se aumentar o diagnóstico pré-natal e melhorar o manejo perinatal destes fetos. Por ser uma técnica relativamente simples e reprodutível, e não necessariamente necessitar da US3D para a sua obtenção, acredita-se que possa ser incorporada à ultrassonografia morfológica de segundo trimestre, como uma forma de se tentar diagnosticar mais precocemente e com maior precisão as disgenesias do CC fetal.

\section{Referências}

1. Fratelli N, Papageorghiou AT, Prefumo F, Bakalis S, Homfray $T$, Thilaganathan B. Outcome of prenatally diagnosed agenesis of the corpus callosum. Prenat Diagn. 2007;27(6):512-7.

2. Schell-Apacik CC, Wagner K, Bihler M, Ertl-Wagner B, Heirinch $U$, Klopocki $E$, et al. Agenesis and dysgenesis of the corpus callosum: clinical, genetic and neuroimaging findings in a series of 41 patients. Am J Med Genet A. 2008;146A(19):2501-11.

3. Lerman-Sagie T, Ben-Sira L, Achiron R, Schreiber L, Herman G, Lev $D$, et al. Thick fetal corpus callosum: an ominous sign? Ultrasound Obstet Gynecol. 2009;34(1):55-61.
4. Volpe P, Paladini D, Resta M, Stanziano A, Salvatore M, Quarantelli $M$, et al. Characteristics, associations and outcome of partial agenesis of the corpus callosum in the fetus. Ultrasound Obstet Gynecol. 2006;27(5):509-16.

5. Babcock DS. The normal, absent, and abnormal corpus callosum: sonographic findings. Radiology. 1984;151 (2):449-53.

6. Pilu G, Segata M, Ghi T, Carletti A, Perolo A, Santini D, et al. Diagnosis of midline anomalies of the fetal brain with the three-dimensional median view. Ultrasound Obstet Gynecol. 2006;27(5):522-9. 
7. Ghi T, Carletti A, Contro E, Cera E, Falco P, Tagliavini G, et al. Prenatal diagnosis and outcome of partial agenesis and hypoplasia of the corpus callosum. Ultrasound Obstet Gynecol. 2010;35(1): 35-41.

8. Gonçalves LF, Lee W, Espinoza J, Romero R. Three- and 4-dimensional ultrasound in obstetric practice: does it help? J Ultrasound Med. 2005;24(12): 1599-624.

9. Pilu G, Ghi T, Carletti A, Segata M, Perolo A, Rizzo N. Threedimensional ultrasound examination of the fetal central nervous system. Ultrasound Obstet Gynecol. 2007;30(2):233-45.

10. Correa FF, Lara C, Bellver J, Remohi J, Pellicer A, Serra V. Examination of the fetal brain by transabdominal three-dimensional ultrasound: potential for routine neurosonographic studies. Ultrasound Obstet Gynecol. 2006;27(5):503-8.

11. Plasencia W, Dagklis T, Borenstein $M$, Csapo B, Nicolaides KH. Assessment of the corpus callosum at 20-24 weeks' gestation by three-dimensional ultrasound examination. Ultrasound Obstet Gynecol. 2007;30(2):169-72.

12. Vinãls F, Muñoz M, Naveas R, Giuliano A. Transfrontal threedimensional visualization of midline cerebral structures. Ultrasound Obstet Gynecol. 2007;30(2):162-8.

13. Bornstein E, Monteagudo A, Santos R, Keller SM, Timor-Tritsch IE. A systematic technique using 3-dimensional ultrasound provides a simple and reproducible mode to evaluate the corpus callosum. Am J Obstet Gynecol. 2010;202(2):201.e1-5.

14. Hadlock FP, Harrist RB, Martinez-Poyer J. In utero analysis of fetal growth: a sonographic weight standard. Radiology. $1991 ; 181(1): 129-33$.

15. Moore TR, Cayle JE. The amniotic fluid index in normal human pregnancy. Am J Obstet Gynecol. 1990;162(5): $1168-73$.
16. Bland JM, Altman DG. Statistical methods for assessing agreement between two methods of clinical measurement. Lancet. 1986; 1 (8476):307-10.

17. Kara A, Hakan Ozturk A, Kurtoglu Z, Umit Talas D, Aktekin M, Saygili $M$, et al. Morphometric comparison of the human corpus callosum in deaf and hearing subjects: an MRI study. J Neuroradiol. 2006;33(3):158-63.

18. Luders E, Narr KL, Hamilton LS, Phillips OR, Thompson PM, Valle JS, et al. Decreased callosal thickness in attention-deficit/hyperactivity disorder. Biol Psychiatry. 2009;65(1):84-8.

19. Georgy BA, Hesselink JR, Jernigan TL. MR imaging of the corpus callosum. Am J Roentgenol. 1993; 160(5):949-55.

20. Atlas SW, Shkolnik A, Naidich TP. Sonographic recognition of agenesis of the corpus callosum. Am J Roentgenol. 1985; 145(1): 167-73.

21. Gebarski SS, Gebarski KS, Bowerman RA, Silver TM. Agenesis of the corpus callosum: sonographic features. Radiology. 1984;151(2):443-8.

22. Malinger $\mathrm{G}$, Zakut $\mathrm{H}$. The corpus callosum: normal fetal development as shown by transvaginal sonography. Am J Roentgenol. 1993; 161(5):1041-3.

23. Achiron R, Achiron A. Development of the human fetal corpus callosum: a high-resolution, cross-sectional sonographic study. Ultrasound Obstet Gynecol. 2001;18(4):343-7.

24. Zhang HC, Yang J, Chen ZP, Ma XY. Sonographic study of the development of fetal corpus callosum in a Chinese population. J Clin Ultrasound. 2009;37(2):75-7.

25. Visentin A, Pilu G, Falco P, Bovicelli L. The transfrontal view: a new approach to the visualization of the midline cerebral structures. J Ultrasound Med. $2001 ; 20(4): 329-33$. 